\title{
Estimación del potencial de los trenes de media y alta velocidad en México con base en la demanda de pasajeros aéreos
}

\author{
Estimation of the Potential of Medium and High Speed Trains in Mexico \\ Considering the Air Passenger Demand
}

\author{
Herrera-García Alfonso \\ Coordinación de Integración del Transporte \\ Instituto Mexicano del Transporte, Querétaro \\ Correo:aherrera@imt.mx
}

\author{
Sánchez-López Orlando \\ División de Estudios de Posgrado e Investigación \\ Facultad de Ingeniería \\ Instituto Tecnológico de Orizaba, Veracruz \\ Correo:orlando_sanchez_lopez@yahoo.com.mx
}

Información del artículo: recibido: diciembre de 2013, reevaluado: marzo 2014, aceptado: junio 2014

\section{Resumen}

Se ha detectado que en trayectos aéreos menores a 500 kilómetros el transporte ferroviario de media y alta velocidad tiene ventajas sobre el servicio aéreo. El propósito de esta investigación fue estimar el potencial de estos servicios de trenes en México, con base exclusivamente en la demanda que generan los pasajeros del servicio aéreo. Para el trazado preliminar de las rutas con potencial, que sirvió como soporte para algunas estimaciones, se utilizó un sistema de información geográfica. Se determinó que existe un potencial de diez rutas ferroviarias para los trenes de media velocidad y de ocho para los de alta. En estas rutas se podrían movilizar más de cuatro millones de pasajeros anualmente. Además, se detectó que estos nuevos servicios brindarían otros beneficios, por ejemplo, en términos ambientales los trenes de media velocidad podrían reducir anualmente la emisión de aproximadamente $213 \mathrm{kt}$ de $\mathrm{CO}_{2}$, ó $248 \mathrm{kt}$ en el caso de los de alta. También se determinó que con estos servicios se lograría aplazar la saturación total del Aeropuerto Internacional de la Ciudad de México cuatro años más; y que las rutas equipadas con servicio de alta velocidad requieren en promedio inversiones 27 veces mayores a las de los servicios de media velocidad.

\section{Descriptores:}

- alta velocidad

- media velocidad

- pasajero aéreo

- sistema de información geográfica

- tren 


\begin{abstract}
It has been detected that in air short-haul up to 500 kilometers distances, the middle and high-speed train services have advantages over the air service. The objective of this research was to estimate the potential of these train services in Mexico considering exclusively the demand that it is generated by the air service. For the preliminary outline of the potential routes, that supports some estimates, a geographic information system was used. It was determined that there is potential of ten train routes for middle-speed trains and eight train routes for high-speed trains. It is possible to transport in these routes more than four million people annually. In addition, it was detected that these new services would provide other benefits, for instance, in environmental terms the middle and high-speed trains could reduce annually the emission of $213 \mathrm{kt}$ and $248 \mathrm{kt}$ of $\mathrm{CO}_{2}$ respectively. Also, it was determined that these services could postpone four more years the complete saturation of the Mexico City International Airport; and that the routes equipped for the highspeed service require 27 times higher investments than the middle-speed service
\end{abstract}

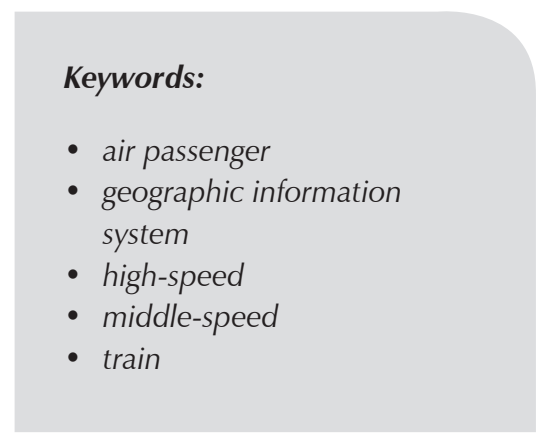

\section{Introducción}

El objetivo general de este trabajo fue determinar el potencial de la utilización de trenes de media y alta velocidad en México, con base en la demanda de los flujos de pasajeros aéreos en recorridos menores a 500 kilómetros. El análisis se centró en los flujos recientes de pasajeros aéreos de corto itinerario. Se consideraron tanto flujos nacionales, como internacionales; además, se tomó en cuenta la infraestructura ferroviaria actual y la orografía de las rutas potenciales.

Los objetivos particulares fueron: cuantificar los volúmenes de pasajeros potenciales, determinar las rutas de estos flujos, estimar la inversión aproximada de cada ruta potencial y cuantificar algunos beneficios de la implementación de las rutas con potencial ferroviario.

El Plan Nacional de Desarrollo (PND) 2013-2018 (GR, 2013) establece que el Sistema Ferroviario Nacional está compuesto de $26,727 \mathrm{~km}$ de vías férreas, de los cuales $18 \%$ está fuera de operación. Por su parte, el Sistema Aeroportuario Nacional se compone de 60 aeropuertos que transportan alrededor de 80 millones de pasajeros al año. De estos, 17 concentran $86 \%$ del tránsito de pasajeros. Algunos de los principales retos que enfrenta actualmente el sector transporte son: el estado físico de las vías y la falta de doble vía en sitios estratégicos, lo cual limita la velocidad del sistema ferroviario; la gran disparidad en el uso de los aeropuertos, pues muchos se subutilizan mientras que algunos se encuentran saturados; por último, la falta de infraestructura aeroportuaria adecuada en el centro del país que limita la capacidad de México para establecerse como el principal centro de conexión de pasajeros y carga de Latinoamérica. Por otra parte, en el aspecto ambiental la estrategia 4.4.3 del PND establece la necesidad de forta- lecer la política nacional de cambio climático y cuidado al medio ambiente para transitar hacia una economía competitiva, sustentable, resiliente y de bajo carbono. Para lo anterior, dentro de las líneas de acción se señala acelerar el tránsito hacia un desarrollo bajo en carbono en los sectores productivos primarios, industriales y de la construcción, así como en los servicios urbanos, turísticos y de transporte. En relación con la infraestructura de transporte el objetivo 4.9 del PND establece contar con una infraestructura de transporte que se refleje en menores costos para realizar la actividad económica, para ello, la estrategia 4.9.1 establece modernizar, ampliar y conservar la infraestructura de los diferentes modos de transporte, así como mejorar su conectividad bajo criterios estratégicos y de eficiencia. Algunas líneas de acción señalan para el sector ferroviario, construir nuevos tramos ferroviarios, libramientos, acortamientos y relocalización de vías férreas que permitan conectar nodos del Sistema Nacional de Plataformas Logísticas; y para el sector aeroportuario, dar una respuesta de largo plazo a la demanda creciente de servicios aeroportuarios en el Valle de México y centro del país.

De acuerdo con las características de velocidad media y máxima de circulación, un tren de media velocidad es aquel que alcanza una velocidad entre los $160 \mathrm{y}$ los $200 \mathrm{~km} / \mathrm{h}$ (Fernandez, 2006). Dentro de este rango de velocidades, los ferrocarriles interurbanos (Inter-city) forman parte de esta clasificación. De acuerdo con el Servicio de Asesoramiento para Infraestructura Pública y Privada del Banco Mundial, los trenes interurbanos de pasajeros, usualmente son impulsados por locomotoras eléctricas, diesel o duales (diesel-eléctricas). Las velocidades máximas de estos equipos rodantes son de aproximadamente $200 \mathrm{~km} / \mathrm{h}$, sobre vías férreas específi- 
camente actualizadas para tales efectos (PPIAF, 2011). Por sus características, un tren interurbano es un tren expreso con paradas limitadas y vagones cómodos para viajes de media y larga distancia. En muchos países europeos, el término "Intercity" o "Inter-City" es un sinónimo de una red de ferrocarriles de intervalo regular, que cumple con ciertos criterios de rapidez y comodidad, y tiene menos paradas que los trenes locales y de cercanías (Muriel, 2011).

El tren de alta velocidad (TAV), según la International Union of Railways (http://www.uic.org/spip. php?article971), es el que alcanza velocidades superiores a $200 \mathrm{~km} / \mathrm{h}$ sobre líneas existentes actualizadas, ó $250 \mathrm{~km} / \mathrm{h}$ sobre líneas específicamente diseñadas para este servicio. La alta velocidad es una combinación de todos los elementos que constituyen dicho sistema, es decir la infraestructura, formada por nuevas líneas férreas o líneas acondicionadas, el material rodante y las condiciones de operación. En todos los casos se trata de equipos rodantes y vías férreas especializados, dado que las velocidades alcanzadas requieren técnicas de alta precisión y calidad. Además, su elevada velocidad les permite competir con el transporte aéreo para distancias medias y largas (Gutiérrez, 2004). Algunos estudios señalan que la reducción de la demanda aeroportuaria mediante el cambio del tránsito aéreo de corto itinerario, hasta 500 kilómetros de distancia, hacia otros modos de transporte de alta velocidad, como el $\mathrm{TAV}$, puede generar beneficios significativos en la reducción de la congestión aeroportuaria. Sin embargo, esta solución requiere inversiones significativas en infraestructura y equipos. En distancias de 500 a 800 kilómetros, el transporte ferroviario ofrece beneficios significativos en ahorro de tiempo de transporte, costos de operación, consumo energético y en la reducción de emisiones de gases contaminantes y ruido (European Commission, 1998). Los beneficios dependen principalmente de dos factores: la distancia entre las zonas origen-destino y la accesibilidad (orografía) de la ruta. El servicio de trenes de alta velocidad en distancias de aproximadamente $500 \mathrm{~km}$ ofrece una mejor ventaja relativa en comparación con el servicio de transporte aéreo de pasajeros (Widmer y Axhausen, 2001). El efecto de implementar ferrocarriles de alta velocidad para el transporte masivo de pasajeros contribuye a un transporte más sostenible en comparación con los viajes carreteros y aéreos de larga distancia. Cuando el tren de alta velocidad (TGV, Train à Grande Vitesse) fue abierto en 1981 entre París y Lyon (Francia), con un tiempo de recorrido competitivo con el modo aéreo, virtualmente todo el tránsito de pasajeros aéreos entre ambas ciudades cambió al sistema de tren (Veldhuis, 1990). Si tales enlaces con trenes de alta velocidad se desarrollan para conectar a los principales aeropuertos, estos pueden servir como un excelente alimentador para los flujos de transporte aéreo. Los trenes de alta velocidad son una opción viable a considerar en los corredores de tránsito de alta densidad. La principal restricción para su implementación es el requerimiento de un capital masivo de inversión. Estudios del impacto de los TAV en los aeropuertos europeos indican su alto potencial para reemplazar a los vuelos alimentadores y la capacidad para reducir el problema de congestión en los aeropuertos (Widmer y Hidber, 2000).

\section{Desarrollo}

El método consistió en determinar inicialmente todos los pares de aeropuertos con operaciones aéreas en 2012, que tuvieran una separación igual o menor a 500 kilómetros. Después, se estimaron los flujos de pasajeros en estos pares para ese año. Posteriormente, se estableció el flujo mínimo a considerar de acuerdo con las capacidades de los equipos ferroviarios comerciales, tanto para trenes de media como de alta velocidad. Enseguida, se realizaron pronósticos de los flujos de pasajeros con potencial para determinar sus tendencias y estimar su crecimiento para el periodo 2013-2016. Con base en un sistema de información geográfica se realizó el trazo preliminar de las rutas ferroviarias con potencial, considerando la orografía y la infraestructura actual. Debido a sus características operativas los ferrocarriles ofrecen beneficios ambientales en comparación con el modo aéreo, ya que generan una menor cantidad de gases contaminantes por cada pasajero-kilómetro transportado. Tomando en cuenta esto, se estimaron los beneficios ambientales de las nuevas rutas ferroviarias respecto al transporte aéreo. Adicionalmente, se estimó el efecto que se presentaría al disminuir la demanda del Aeropuerto Internacional de la Ciudad de México, como consecuencia del traslado de parte de su demanda al modo ferroviario, para ello se utilizó un modelo de simulación.

Determinación de los flujos de pasajeros por pares de aeropuertos en 2012, con trayectos iguales o menores a 500 kilómetros

Para estimar la distancia de estos trayectos se calculó la distancia ortodrómica entre cada par de aeropuertos considerados. Mediante el procesamiento de las bases de datos de la Dirección General de Aeronáutica Civil (DGAC), para el año 2012, se determinó que 67 aeropuertos mexicanos tuvieron operaciones aéreas y se de- 
tectaron 873 rutas aéreas (432 nacionales y 441 internacionales). Con objeto de calcular las distancias ortodrómicas entre cada par de aeropuertos se utilizaron las siguientes ecuaciones:

$d=\mathrm{r} \Delta \hat{\sigma}$

donde:

$d=$ distancia entre cada par de aeropuertos origen y destino,

$r=$ radio de la tierra (6,372.8 kilómetros) $\mathrm{y}$

$\Delta \widehat{\sigma}=$ ángulo central, en radianes, formado por cada par de aeropuertos.

Además:

$\Delta \hat{\sigma}=\arctan \left(\frac{\sqrt{\left(\cos \varnothing_{f} \operatorname{sen} \Delta \lambda\right)^{2}+\left(\cos \varnothing_{S} \operatorname{sen} \varnothing_{f}-\operatorname{sen} \varnothing_{S} \cos \varnothing_{f} \cos \Delta \lambda\right)^{2}}}{\operatorname{sen} \varnothing_{S} \operatorname{sen} \varnothing_{f}+\cos \varnothing_{S} \cos \varnothing_{f} \cos \Delta \lambda}\right)$

donde $\varphi_{s^{\prime}} \lambda_{s^{\prime}}: \varphi_{f^{\prime}} \lambda_{f}$ son la latitud y longitud geográfica de cada aeropuerto origen y destino respectivamente y $\Delta \varphi, \Delta \lambda$ son las diferencias de esos valores.

Las coordenadas geográficas de los 67 aeropuertos en México se obtuvieron de la Publicación de Información Aeronáutica (SENEAM, 2012). Cabe señalar que para esta estimación se consideraron las coordenadas del punto de referencia de cada aeropuerto (ARP, Airport Reference Point). Con este procedimiento se calcularon las distancias ortodrómicas de las 873 rutas detectadas. Como resultado se identificaron 150 pares de aeropuertos con distancias menores o iguales a 500 kilómetros. En la tabla 1 se presentan los primeros doce pares de aeropuertos en función de los flujos de pasajeros en cada par, ordenados de mayor a menor.
Selección de los equipos ferroviarios comerciales

Se estableció que el flujo mínimo con potencial ferroviario será aquel que permita al menos un viaje diario en ambos sentidos de los pares de aeropuertos detectados, utilizando equipos ferroviarios comerciales. Con objeto de establecer la magnitud de estos flujos mínimos, se realizó una búsqueda documental para determinar los trenes comerciales de media y alta velocidad que operan en la actualidad, con los valores más bajos en capacidad. En el caso del equipo rodante de media velocidad (entre 160 y $200 \mathrm{~km} / \mathrm{h}$ ) se consideraron trenes italianos (como el ETR 401), trenes con sistema pendular (como el tren sueco X-2000), los trenes españoles Talgo, y el tren británico IC (Intercity) 225. Se encontró que el equipo con menor capacidad comercial de pasajeros corresponde al tren español con sistema pendular de la compañía Renfe Serie 594. Este equipo lo fabrica CAF y Adtrantz y puede desarrollar una velocidad máxima de $200 \mathrm{~km} / \mathrm{h}$, tiene una potencia de $1,200 \mathrm{~kW}$, un peso de 97 toneladas y una capacidad de 126 pasajeros en clase única (http://suite101.net/article/automotor-diesel-594de-renfe-un-giro-en-los-trenes-regionales-a59151\#. U3un8tJ5OSo).

En el caso de los equipos de alta velocidad (entre 200 y $350 \mathrm{~km} / \mathrm{h}$ ), se evaluaron los trenes TGV-Sud-EstAVE de España; los ICE (Inter-City Express) de Alemania; el ETR 500 italiano; los TGV-Duplex, TGVAtlantique, TGV-Nord y TGV-Thalys de Francia; el HST británico y los trenes del Eurostar Group. En este caso, el equipo con la mínima capacidad correspondió al tren con sistema pendular de la compañía Renfe Serie 490. Este equipo lo fabrica el consorcio GEC-AlsthomFIAT, puede alcanzar una velocidad máxima de 220

\begin{tabular}{|c|c|c|c|}
\hline \multicolumn{2}{|c|}{ Pares de aeropuertos } & $\begin{array}{l}\text { Flujo anual de } \\
\text { pasajeros }\end{array}$ & $\begin{array}{c}\text { Distancia entre } \\
\text { aeropuertos }(\mathrm{km})\end{array}$ \\
\hline Guadalajara & México & $2,024,787$ & 459.3 \\
\hline México & Veracruz & 453,993 & 304.5 \\
\hline México & Tampico & 422,334 & 341.3 \\
\hline México & Oaxaca & 343,586 & 367.3 \\
\hline Acapulco & México & 304,395 & 306.6 \\
\hline Del Bajío & México & 242,221 & 304.8 \\
\hline México & Zihuatanejo & 217,476 & 324.2 \\
\hline Aguascalientes & México & 176,816 & 421.8 \\
\hline México & $\begin{array}{l}\text { San Luis } \\
\text { Potosí }\end{array}$ & 148,260 & 368.2 \\
\hline México & Minatitlán & 129,463 & 496.2 \\
\hline Guadalajara & Toluca & 98,776 & 413.2 \\
\hline Acapulco & Toluca & 96,078 & 287.6 \\
\hline
\end{tabular}

Tabla 1. Principales pares de aeropuertos con base en los flujos de pasajeros, con separaciones iguales o menores a 500 kilómetros, para el año 2012

Fuente: elaboración propia con base en estadísticas de la Dirección General de Aeronáutica Civil (DGAC) 
$\mathrm{km} / \mathrm{h}$, tiene una potencia de 1,960 kW, un peso de 159 toneladas y una capacidad de 160 plazas (http://www. ferropedia.es/wiki/Renfe_Serie_490). Sin embargo, para las rutas de alta densidad de pasajeros resulta recomendable el equipo Renfe Serie 112, que es un tren fabricado por Talgo en asociación con Bombardier. Es un equipo ligero, con coches articulados, pendulación natural y centro de gravedad bajo, que puede alcanzar velocidades de $350 \mathrm{~km} / \mathrm{h}$. Cada tren consta de 12 coches Talgo-350, con una capacidad total de 365 plazas (http:// www.ferropedia.es/wiki/Renfe_Serie_112).

\section{Estimación de los flujos de pasajeros ferroviarios mínimos}

De acuerdo con las capacidades de los equipos ferroviarios seleccionados en el inciso anterior y considerando que operan los 365 días del año, con al menos un viaje en cada sentido (viaje redondo), en la tabla 2 se muestra la estimación de los flujos anuales mínimos de pasajeros para los trenes de media y alta velocidad.

Tabla 2. Estimación de flujo de pasajeros ferroviarios mínimo anual

\begin{tabular}{lcc}
\hline $\begin{array}{c}\text { Tipo de servicio de } \\
\text { transporte de pasajeros }\end{array}$ & $\begin{array}{c}\text { Capacidad del } \\
\text { equipo (pasajeros) }\end{array}$ & $\begin{array}{c}\text { Flujo anual } \\
\text { (pasajeros) }\end{array}$ \\
\hline Tren de media velocidad & 126 & 91,980 \\
Tren de alta velocidad & 160 & 116,800 \\
\hline Fuente: elaboración propia & &
\end{tabular}

De acuerdo con estos valores, para el año 2012 todos los pares señalados en la tabla 1, serían candidatos para trenes de media velocidad y los 10 primeros para el de alta velocidad. Sin embargo, falta revisar sus tendencias para asegurar su viabilidad en el futuro, esto se presenta en el siguiente inciso.

Pronósticos de los flujos de pasajeros en las rutas ferroviarias con potencial

Con base en series estadísticas de ocho años (2005 a 2012) de los flujos de pasajeros registrados por la DGAC, en los pares de aeropuertos señalados en la tabla 1 , se realizaron pronósticos para determinar cómo sería su comportamiento futuro. Esto con objeto de asegurar que dichos flujos no tienden a decrecer, debido a que si algún par tuviera una tendencia decreciente, la ruta definida por este no sería viable. Para determinar estas tendencias no es suficiente con observar gráficamente su comportamiento, por lo que además, para cada una de las series de datos de los pares señalados en el inciso anterior, se realizaron pruebas de hipótesis
(Pearson) considerando un nivel de confianza de 95\%. Las hipótesis fueron las siguientes:

$\mathrm{H}_{0}: \rho=0$, hipótesis de que no existe tendencia en los flujos de pasajeros.

$H_{\alpha}: \rho \neq 0$, hipótesis de que sí existe tendencia en los flujos de pasajeros.

Por un lado, cuando la prueba de hipótesis señaló que los datos no tenían tendencia, se buscaron modelos acotados a pronósticos estacionarios, precisamente porque no se presenta la característica de tendencia. En este caso, los modelos considerados fueron los siguientes: promedios móviles de longitud k (PM), promedios móviles ponderados (PMP) y suavización exponencial (S. EXP.). Por otra parte, en los casos que sí se presentó tendencia, pero fue decreciente, se descartó el par respectivo debido a que se consideró que no sería rentable. Sin embargo, cuando la tendencia fue creciente, se realizó una búsqueda acotada a los siguientes modelos de pronósticos no estacionarios: modelo lineal de mínimos cuadrados (Lineal M2), modelo cuadrático de mínimos cuadrados (Cuadrático M2), modelo de ajuste logístico Gompertz (GOMP) y suavización exponencial doble (SE. DOBLE).

Después, con los datos ajustados que se obtuvieron de estos modelos, se estimaron los residuales (diferencias entre los datos reales y los valores ajustados correspondientes). El siguiente paso consistió en analizarlos mediante pruebas de normalidad e independencia. Si los residuales se comportaban de manera normal e independiente, los modelos se consideraron como buenos candidatos para pronosticar la serie temporal. Para el análisis de normalidad se aplicó la prueba AndersonDarling, y para el de independencia, la prueba de rachas, en ambos casos considerando un alfa de $5 \%$. Cuando ambas pruebas fueron estadísticamente satisfactorias para los modelos se seleccionó el mejor de ellos, con base en dos medidas de desempeño, la desviación media absoluta (MAD) y el porcentaje de error medio absoluto (MAPE). En cada caso el modelo utilizado finalmente para realizar los pronósticos fue el que presentó los valores más bajos en dichas medidas de desempeño. Debido a su tendencia decreciente, dos rutas aéreas fueron descartadas de los análisis posteriores, este es el caso de los pares de aeropuertos Acapulco-México y México-Zihuatanejo. Un resumen de este análisis se presenta en la tabla 3.

En la tabla 4 se presentan los pronósticos obtenidos para el año 2013 con base en el procedimiento señalado. También, se indica para qué tipo de servicio se cumpliría con el requisito de flujo mínimo de pasajeros. Se ob- 
serva que para los diez pares de esta tabla existe potencial para el desarrollo de los trenes de media velocidad, y solo para los primeros ocho, para los de alta velocidad. También, con los modelos obtenidos antes se realizaron los pronósticos para los años 2014, 2015 y 2016.

\section{Trazo preliminar de las rutas ferroviarias con potencial}

Para este trazo se utilizó un sistema de información geográfica, considerando la orografía y la infraestructura ferroviaria existente. En las figuras 1 y 2 se resumen los resultados obtenidos para los trenes de media y alta velocidad, respectivamente. Las bases de datos geográficos de la red mexicana ferroviaria actual y de las características orográficas fueron proporcionadas por el Instituto Mexicano del Transporte (IMT).

Se debe observar cómo en el caso de la red para trenes de media velocidad se ocupa en buena medida infraestructura existente, excepto en la ruta TolucaAcapulco y gran parte de la ruta México-Tampico, que es infraestructura nueva. Por su parte, en la red ferroviaria de alta velocidad, toda la infraestructura es nueva debido a que se requiere un trazo más recto y con mayores especificaciones técnicas. En las figuras se presentan además del trazo preliminar de la red ferroviaria existente y/o nueva, la concentración de población, los ríos, las elevaciones y las rutas aéreas asociadas. Se observa cómo los trazos preliminares de las rutas ferroviarias de media y alta velocidad tienen relación con las concentraciones de población. La capa de información georreferenciada de la población para el año 2010, se obtuvo del Instituto Nacional de Estadística y Geografía (INEGI, http://www.inegi.org.mx/).

Este trazo preliminar de las rutas ferroviarias con potencial, también incluyó la determinación de su perfil longitudinal, pendiente y curvatura para definir el tipo de terreno sobre el que está o estará construida (plano, lomerío o montañoso). Cabe señalar que $80 \%$ de las rutas ferroviarias analizadas tienen un perfil de terreno tipo montañoso, este dato es de gran utilidad para estimar el costo de la infraestructura ferroviaria requerida.

\section{Estimación de las inversiones requeridas para implementar los servicios de trenes}

Trenes de media velocidad

De acuerdo con lo establecido, en el caso de los trenes de media velocidad se consideró el tren Renfe serie 594, con unidades en clase única con una capacidad de 126 pasajeros. Estos vehículos son la única serie de Renfe que pueden formar composiciones de hasta cinco unidades, logrando una capacidad máxima de 630 pasajeros. En una composición múltiple las unidades son comandadas por una sola cabina de conducción. Están construidas en aleación ligera, con un sistema que permite el libre tránsito a los viajeros entre las diferentes unidades, una vez acopladas. En 2006, este diseño tenía un costo por unidad de 5.35 millones de euros (http://

Tabla 3. Resumen de los modelos a utilizar para realizar los pronósticos

\begin{tabular}{lcccc}
\hline Pares de aeropuertos & $\begin{array}{c}\text { Existe tendencia en los } \\
\text { flujos de pasajeros }\end{array}$ & Mejor modelo & MAD & MAPE \\
\hline Guadalajara - México & Sí (creciente) & Cuadrático M2 & $62,093.41$ & 0.03627 \\
México - Veracruz & No & PM de longitud 2k & $5,869,966.67$ & 11.93 \\
México - Tampico & Sí (creciente) & Cuadrático M2 & $16,783.16$ & 0.05335 \\
México - Oaxaca & No & S. EXP & $3,567,941.58$ & 10.89 \\
Acapulco -México & Sí (decreciente) & Se descartó & No aplica & No aplica \\
Del Bajío - México & No & S. EXP. & $995,537.67$ & 4.17 \\
México - Zihuatanejo & Sí (decreciente) & Se descartó & No aplica & No aplica \\
Aguascalientes - Méx. & No & S. EXP. & $1,732,568.75$ & 9.79 \\
México - SLP & No & S. EXP. & $1,100,008.98$ & 8.24 \\
México - Minatitlán & No & PMP de longitud 2 & $2,088,123.33$ & 14.04 \\
Guadalajara - Toluca & No & PMP de longitud 2 & $10,641,135.00$ & 48.61 \\
Acapulco - Toluca & No & S. EXP. & $1,736,114.38$ & 18.53 \\
\hline Fuente: elaboración propia & & & &
\end{tabular}


Tabla 4. Pronósticos de los flujos de pasajeros para el año 2013

\begin{tabular}{lccc}
\hline Pares de aeropuertos & $\begin{array}{c}\text { Pronóstico de flujo de pasajeros } \\
\text { anual para 2013 }\end{array}$ & $\begin{array}{c}\text { Servicio de media } \\
\text { velocidad }\end{array}$ & $\begin{array}{c}\text { Servicio de alta } \\
\text { velocidad }\end{array}$ \\
\hline Guadalajara - México & $2,051,136$ & Aplica & Aplica \\
México - Veracruz & 475,485 & Aplica & Aplica \\
México - Tampico & 449,085 & Aplica & Aplica \\
México - Oaxaca & 344,989 & Aplica & Aplica \\
Del Bajío - México & 234,476 & Aplica & Aplica \\
Aguascalientes - Méx. & 170,473 & Aplica & Aplica \\
Guadalajara - Toluca & 137,816 & Aplica & Aplica \\
México - SLP & 137,670 & Aplica & Aplica \\
Acapulco - Toluca & 115,504 & Aplica & No aplica \\
México - Minatitlán & 96,271 & Aplica & No aplica \\
\hline
\end{tabular}

Fuente: elaboración propia
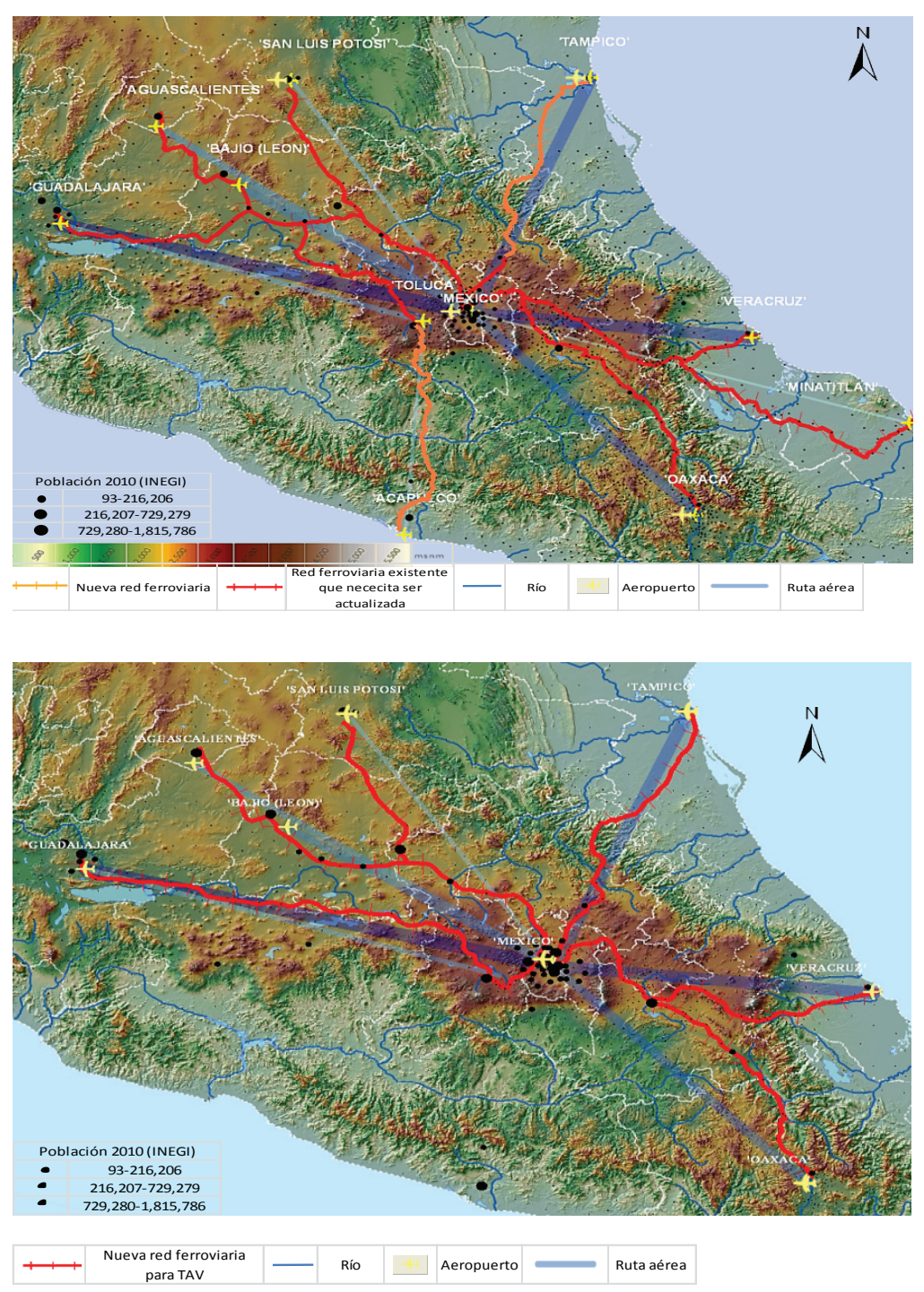

Figura 1. Red ferroviaria con potencial para trenes de media velocidad (Fuente: elaboración propia)

Figura 2. Red ferroviaria con potencial para trenes de alta velocidad (Fuente: elaboración propia) 
www.ferropedia.es/wiki/Renfe_Serie_594). Considerando la tasa de variación interanual del índice de precios industriales de España (http://www.idescat.cat/ novetats/?id=1643\&lang=es y http://www.ine.es/prensa/ipri_prensa.htm), se estimó que el valor de estos equipos en 2014 es de 6.366 millones de euros, equivalentes a 114.847 millones de pesos (considerando un tipo de cambio de 18.04 pesos por euro, (http://www. banxico.org.mx/).

En función de la demanda promedio diaria de cada ruta, su longitud y considerando una velocidad de servicio promedio de 160 kilómetros/hora, se determinó el número de unidades requeridas en cada ruta. Los resultados se presentan en la tabla 5, incluyendo el costo total de los equipos estimados.

Para la operación de estos materiales rodantes se requiere una actualización de la infraestructura ferroviaria, pero aprovechando los puentes y túneles existentes. La excepción son las rutas ferroviarias de AcapulcoToluca y México-Tampico, en las que se realizó un trazo nuevo de vía, mediante un sistema de información geográfica, bordeando sistemas montañosos y considerando la construcción de los puentes necesarios para el cruce de ríos. Con objeto de estimar el costo de la infraestructura ferroviaria asociada con la operación de los trenes de media velocidad, se utilizaron los costos promedio establecidos por Martínez et al. (2012) y las tasas disponibles de inflación para 2013 y 2014 (http:// www.bancodemexico.gob.mx/portal-inflacion/index. html). Además, para cada ruta se requirió conocer su longitud y tipo de terreno. El costo de los puentes ferroviarios (2014) para soportar velocidades de hasta 220 $\mathrm{km} / \mathrm{h}$ es de 38.67 millones de pesos por kilómetro (con base en la tasa de variación interanual del índice de precios industriales de España, información del Banco de México y http://www.ferropedia.es/wiki/Costos_de_ construcci\%C3\%B3n_de_infraestructura\#T.C3.BAneles_urbanos). En la tabla 5 se presenta un resumen de los resultados.

\section{Trenes de alta velocidad}

Para el caso de los trenes de alta velocidad en general, se consideró al tren Renfe Serie 490, con una capacidad de 160 pasajeros; y para rutas de alta densidad al Renfe Serie 112, con una capacidad de 365 plazas. En 2006, el primer equipo considerado tenía un costo por unidad de 7.45 millones de euros y el segundo de 24.74 millones de euros (http://www.ferropedia.es/wiki/Renfe_Serie_490yhttp://www.ferro-pedia.es/wiki/Renfe_Serie_112). Nuevamente, tomando en cuenta la tasa de variación interanual del índice de precios industriales de España

Tabla 5. Costos de la implementación de los trenes de media velocidad

\begin{tabular}{|c|c|c|c|c|c|c|c|}
\hline Ruta & $\begin{array}{l}\text { Unidades } \\
\text { de equipo } \\
\text { rodante } \\
\text { requeridas }\end{array}$ & $\begin{array}{l}\text { Costo del } \\
\text { equipo } \\
\text { rodante } \\
\text { (millones } \\
\text { de pesos) } \\
\end{array}$ & $\begin{array}{l}\text { Longitud } \\
\text { de vía } \\
\text { ferroviaria } \\
\text { (kilómetros) }\end{array}$ & $\begin{array}{l}\text { Costo de } \\
\text { infraestructura } \\
\text { ferroviaria, } \\
\text { excepto } \\
\text { puentes } \\
\text { (millones de } \\
\text { pesos) }\end{array}$ & $\begin{array}{l}\text { Longitud total } \\
\text { de puentes } \\
\text { ferroviarios } \\
\text { nuevos para el } \\
\text { cruce de ríos } \\
\text { (kilómetros) }\end{array}$ & $\begin{array}{l}\text { Costo de } \\
\text { los puentes } \\
\text { ferroviarios } \\
\text { nuevos } \\
\text { (millones de } \\
\text { pesos) }\end{array}$ & $\begin{array}{l}\text { Costo total } \\
\text { del equipo } \\
\text { rodante, } \\
\text { infraestructura } \\
\text { y puentes } \\
\text { (millones de } \\
\text { pesos) }\end{array}$ \\
\hline Guadalajara - México & 10 & $1,148.47$ & 593.5 & $7,862.94$ & - & - & $9,011.42$ \\
\hline México - Veracruz & 2 & 229.69 & 410.2 & $5,434.50$ & - & - & $5,664.20$ \\
\hline México - Tampico & 2 & 229.69 & 428.7 & $5,679.60$ & 2.29 & 88.55 & $5,997.84$ \\
\hline México - Oахаса & 2 & 229.69 & 520.3 & $6,893.16$ & - & - & $7,122.85$ \\
\hline Del Bajío - México & 1 & 114.84 & 371.0 & $4,915.17$ & - & - & $5,030.01$ \\
\hline Aguascalientes - Méx. & 1 & 114.84 & 561.6 & $7,440.32$ & - & - & $7,555.17$ \\
\hline Guadalajara - Toluca & 1 & 114.84 & 583.4 & $7,729.13$ & - & - & $7,843.98$ \\
\hline México - SLP & 1 & 114.84 & 415.3 & $5,502.07$ & - & - & $5,616.92$ \\
\hline Acapulco - Toluca & 1 & 114.84 & 378.0 & $5,007.90$ & 1.30 & 50.27 & $5,173.01$ \\
\hline México - Minatitlán & 1 & 114.84 & 673.0 & $8,916.19$ & - & - & $9,031.04$ \\
\hline
\end{tabular}

Fuente: elaboración propia 
y la información del Banco de México, se estimó que el valor de estos equipos en 2014 corresponde a 159.92 y 531.09 millones de pesos, respectivamente.

En función de la demanda promedio diaria de cada ruta, su longitud y considerando una velocidad de servicio de $200 \mathrm{~km} / \mathrm{h}$, se determinó el número de unidades requeridas en cada ruta. Los resultados se presentan en la tabla 6, incluyendo el costo total de los equipos estimados.

Para el servicio de estos equipos rodantes se requiere una nueva infraestructura ferroviaria, con objeto de estimar su costo se realizaron nuevos trazos ferroviarios mediante un sistema de información geográfica, bordeando sistemas montañosos y considerando la construcción de puentes para el cruce de ríos.

Con base en un estudio realizado por Campos et al. (2009) se estimaron los costos de la infraestructura asociada con los trenes de alta velocidad. Este estudio analizó 166 proyectos de trenes de alta velocidad en veinte países, de los cuales 40 se encontraban en operación, 41 estaban en fase de construcción o puesta en funcionamiento, y 85 se hallaban aún en la etapa de planificación. Considerando la información de los proyectos con datos completos (45), el costo promedio de cada kilómetro de línea de alta velocidad era de 17.5 millones de euros, en el año 2005. Este costo incluye los costos de infraestructura, pero no los de planificación y preparación del terreno. Sin embargo, si el análisis se limita a los proyectos en servicio (24), el costo promedio es 18 millones de euros por kilómetro para el año de referencia, este fue el valor utilizado para las siguientes estimaciones. Aplicando la tasa de variación interanual del índice de precios industriales de España, para los años 2006 a 2014, se estimó que el costo promedio de cada kilómetro de infraestructura para 2014 es 22.57 millones de euros, equivalentes a 407.27 millones de pesos. Con el valor unitario anterior y la longitud estimada de cada ruta de alta velocidad, se calculó el costo de la infraestructura respectiva. Por otra parte, el costo en 2009 de los puentes ferroviarios para soportar velocidades de hasta $350 \mathrm{~km} / \mathrm{h}$, era 2.5 millones de euros por kilómetro (http://www.ferropedia.es/wiki/Costos_de_cons trucci\%C3\%B3n_de_infraestructura). Realizando los ajustes necesarios para 2014, esta cifra corresponde a 50.35 millones de pesos por kilómetro. En la tabla 6 se presenta un resumen de los resultados obtenidos.

\section{Beneficios ambientales de las nuevas rutas ferroviarias}

Para estimar los beneficios ambientales, inicialmente se calcularon las emisiones de dióxido de carbono $\left(\mathrm{CO}_{2}\right)$ que se generarían si el servicio fuera brindado por el modo aéreo, y después las emisiones que se generarían por el modo ferroviario, la diferencia entre estos valores determinó el beneficio ambiental de los servicios de trenes.

Para estimar las emisiones de $\mathrm{CO}_{2}$ del modo aéreo se utilizó la calculadora de la Organización de Aviación Civil Internacional (OACI), considerando viajes en clase económica (http://www2.icao.int/en/carbonoffset/ Pages/default.aspx). En relación con las emisiones del modo ferroviario para los trenes de media velocidad se consideró un valor de 27.88 gramos de $\mathrm{CO}_{2}$ de emisio-

Tabla 6. Costos de la implementación de los trenes de alta velocidad

\begin{tabular}{|c|c|c|c|c|c|c|c|c|}
\hline Ruta & $\begin{array}{l}\text { Serie del } \\
\text { equipo } \\
\text { rodante }\end{array}$ & $\begin{array}{l}\text { Unidades } \\
\text { de equipo } \\
\text { rodante } \\
\text { requeridas }\end{array}$ & $\begin{array}{l}\text { Costo del } \\
\text { equipo } \\
\text { rodante } \\
\text { (millones } \\
\text { de pesos) }\end{array}$ & $\begin{array}{l}\text { Longitud de } \\
\text { vía ferroviaria } \\
\text { (kilómetros) }\end{array}$ & $\begin{array}{c}\text { Costo de la } \\
\text { infraestructura } \\
\text { ferroviaria, } \\
\text { excepto puentes } \\
\text { (millones de } \\
\text { pesos) }\end{array}$ & $\begin{array}{l}\text { Longitud total } \\
\text { de puentes } \\
\text { ferroviarios para } \\
\text { el cruce de ríos } \\
\text { (kilómetros) }\end{array}$ & $\begin{array}{c}\text { Costo de } \\
\text { puentes } \\
\text { ferroviarios } \\
\text { (millones de } \\
\text { pesos) }\end{array}$ & $\begin{array}{l}\text { Costo total del } \\
\text { equipo rodante, } \\
\text { infraestructura y } \\
\text { puentes (millones } \\
\text { de pesos) }\end{array}$ \\
\hline Guadalajara - México & 112 & 2 & $1,062.18$ & 549.9 & $223,957.86$ & 0.63 & 31.72 & $225,051.76$ \\
\hline México - Veracruz & 490 & 1 & 159.92 & 423.8 & $172,601.09$ & 0.49 & 24.67 & $172,785.68$ \\
\hline México - Tampico & 490 & 1 & 159.92 & 395.7 & $161,156.80$ & 1.91 & 96.17 & $161,412.89$ \\
\hline México - Oaxaca & 490 & 1 & 159.92 & 471.9 & $192,190.78$ & 0.22 & 11.07 & $192,361.71$ \\
\hline Del Bajío - México & 490 & 1 & 159.92 & 379.6 & $154,599.75$ & 0.19 & 9.56 & $154,769.23$ \\
\hline Aguascalientes - Méx. & 490 & 1 & 159.92 & 504.4 & $205,427.06$ & 0.35 & 17.62 & $205,604.60$ \\
\hline Guadalajara - Toluca & 490 & 1 & 159.92 & 476.4 & $194,023.50$ & 0.63 & 31.72 & $194,215.14$ \\
\hline México - SLP & 490 & 1 & 159.92 & 420.5 & $171,257.10$ & 0.39 & 19.63 & $171,436.65$ \\
\hline
\end{tabular}

Fuente: elaboración propia 
nes por viajero-kilómetro (RENFE, 2008), y para el caso de los trenes de alta velocidad, se consideró que este valor es igual a cero, debido a que se trata de equipos con tracción eléctrica. Los resultados para las 10 rutas con TMV y las 8 de los TAV se muestran en la figura 3. Las líneas punteadas representan las tendencias de sus comportamientos.

\section{Disminución de la saturación en el Aeropuerto Internacional de la Ciudad de México (AICM)}

De las 10 rutas con potencial para los servicios de media velocidad, 8 involucran al AICM, y de las 8 de alta velocidad, siete se relacionan con el AICM. Por lo anterior, es evidente que la entrada en operación de estos servicios podría disminuir la demanda en este aeropuerto, lo que a su vez, retrasaría la saturación total durante algún tiempo. De acuerdo con un estudio previo (Herrera, 2012) se estimó que el AICM alcanzará un nivel de operación crítico en octubre de 2015, cuando opere $80 \%$ de su capacidad máxima (flecha A en la figura 4).

Para estimar en qué medida la implementación de los trenes de media y alta velocidad retrasarían la sa- turación total del AICM, se siguieron algunos pasos. Primero, con base en la metodología señalada, pero ahora aplicada a las operaciones aéreas con potencial para ser transferidas al modo ferroviario, se estimaron estos valores para el periodo 2014-2024. Posteriormente, con base en las estimaciones del crecimiento natural de la demanda de servicio en el AICM (Herrera, 2012), se determinó cuál sería la disminución de la actividad en este aeropuerto al considerar que la demanda de servicio aéreo para las rutas establecidas se cambia totalmente $(100 \%)$ a los trenes de media y alta velocidad (escenario optimista, flecha $\mathrm{D}$ en la figura 4).

También, se consideraron otros dos escenarios (intermedio y pesimista), en donde se supone que únicamente parte de la demanda aérea $(75 \%$ y $50 \%$, respectivamente) se transfiere al servicio de trenes (flechas C y B, respectivamente en la figura 4). Con base en estos cambios de la demanda se estimó cuánto tiempo se diferiría el nivel de operación crítico del AICM.

Por último, con base en un modelo de simulación del AICM (Herrera, 2012) se estimó que al implementar el servicio de trenes de media o alta velocidad, habría

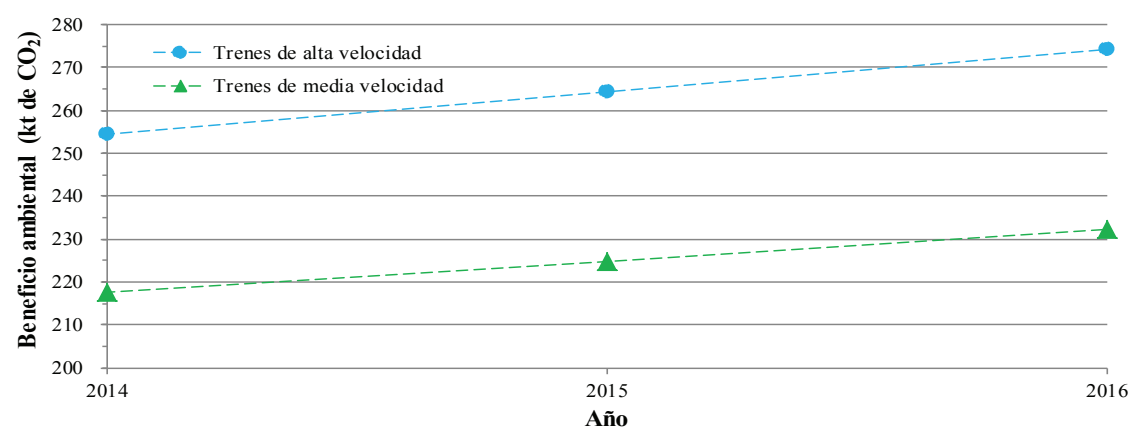

Figura 3. Disminución de las emisiones de $\mathrm{CO}_{2}$ con la operación de trenes, en lugar de aeronaves

Fuente: Elaboración propia

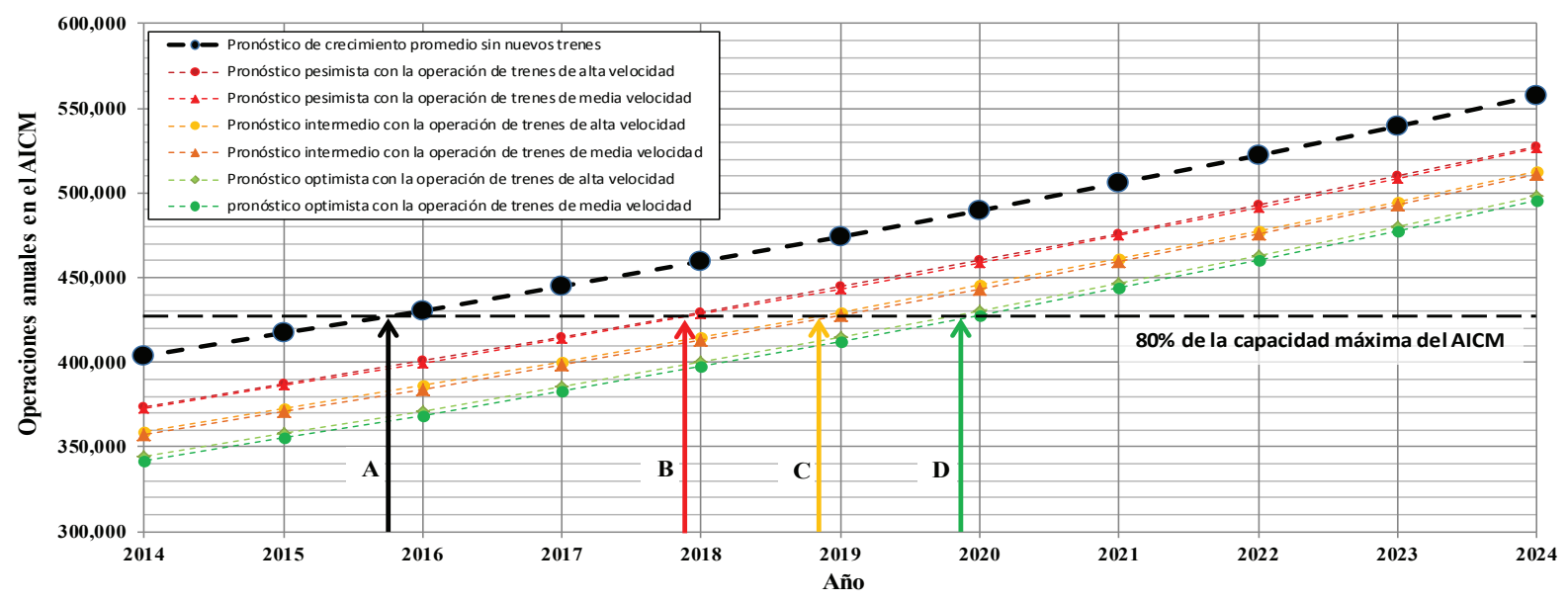

Figura 4. Pronósticos de las operaciones totales anuales en el AICM bajo distintos escenarios (Fuente: elaboración propia) 
una mejora en el servicio de este aeropuerto, dado que habría una disminución en las demoras y líneas de espera en las pistas. Los resultados para octubre de 2015 se presentan en la tabla 7 .

\section{Discusión y análisis de resultados}

Es interesante señalar que dentro de los 150 pares de aeropuertos con una separación menor o igual a 500 kilómetros, también se detectaron aeropuertos internacionales, sin embargo, estos no cumplieron con el requisito del flujo mínimo. No obstante, en el futuro estas rutas podrían crecer lo suficiente para establecer servicios ferroviarios. Dentro de estos pares destacan en Estados Unidos: Monterrey-San Antonio (Texas), Hermosillo-Phoenix (Arizona), y Monterrey-Brownsville (Texas). El desarrollo de estos proyectos implicaría una cooperación binacional. Por otro lado, se debe remarcar la preponderancia del AICM en las rutas con potencial, dado que la Ciudad de México está incluida en la mayoría de las rutas ferroviarias viables de media $(80 \%)$ y alta velocidad $(87.5 \%)$.

Se determinó que con los flujos de pasajeros pronosticados para 2014, existen diez rutas con potencial para el desarrollo de los trenes de media velocidad, y ocho para los de alta velocidad. Para este año de referencia se estima una demanda de 4.3 millones de pasajeros para los trenes de media velocidad, o de 4.1 millones para los de alta. También se observó que cuatro rutas ferroviarias concentran alrededor de $80 \%$ de la demanda. Estas rutas en orden de importancia son: Guadalajara-México, México-Veracruz, México-Tampico y México-Oaxaca. En particular, la primera ruta destaca al concentrar alrededor de $50 \%$ de la demanda potencial.

Con objeto de establecer las prioridades en la implementación de estos servicios sería recomendable aplicar la técnica Electra, la cual analiza diversas alternativas de solución mediante criterios múltiples a través de relaciones binarias de sobreclasificación. En su aplicación al menos se deben considerar los siguientes criterios: pronósticos de pasajeros, costos de operación, costos de infraestructura y beneficio ambiental.

Con base en el análisis de la información aportada por el SIG (figuras 1 y 2) se observa que en general, los orígenes y destinos de las rutas con potencial ferroviario están relacionados con concentraciones de población. En el caso del servicio de media velocidad, las rutas con potencial, en la mayoría de los casos pueden establecerse en forma paralela a la infraestructura actual. Sin embargo, en el caso del trayecto Toluca-Acapulco se trata de un trazo nuevo, situación que también se presenta en buena parte del trayecto México-Tampico. Además, con objeto de no duplicar infraestructura en los casos de rutas comunes o cercanas, se consideró utilizar parte del mismo trayecto y de esta forma disminuir los costos asociados. Por ejemplo, en el caso del servicio de media velocidad, la ruta México-Minatitlán utiliza parte de la ruta México-Veracruz; también, las rutas México-San Luis Potosí, México-Bajío y MéxicoAguascalientes, tienen varios tramos comunes; y por último las rutas Toluca-Guadalajara y México-Guadalajara también, comparten una parte de su infraestructura. En el caso de los trenes de alta velocidad esta situación se presenta en las rutas México-Veracruz y México-Oaxaca; entre las rutas México-San Luis Potosí, México-Bajío y México-Aguascalientes; y en las rutas México-Guadalajara y Toluca-Guadalajara. Otra observación importante es que $80 \%$ de las rutas ferroviarias analizadas tienen un perfil de terreno tipo montañoso, lo que incide desfavorablemente en los costos de la infraestructura requerida.

Los costos de la infraestructura para los servicios de alta velocidad son excesivamente altos, comparados con los de media velocidad. En promedio, en las rutas analizadas son aproximadamente 27 veces mayores. En cuanto a los costos de implementación de estos servicios, las líneas ferroviarias son las que tienen mayor peso en comparación con los equipos rodantes y puentes. En las estimaciones se determinó que estas líneas representan en promedio 96\% y 99.8\%

\begin{tabular}{lccc}
\hline Parámetro considerado & $\begin{array}{c}\text { Sin los trenes } \\
\text { de alta o media } \\
\text { velocidad }\end{array}$ & $\begin{array}{c}\text { Con los } \\
\text { trenes de } \\
\text { alta o media } \\
\text { velocidad }\end{array}$ & $\begin{array}{c}\text { Reducción con los } \\
\text { trenes de alta o } \\
\text { media velocidad }\end{array}$ \\
\hline Demoras promedio (minutos) & 4.5 & 2.5 & 2 \\
Colas promedio (aeronaves) & 4 & 2 & 2 \\
Demoras máximas (minutos) & 20 & 14 & 6 \\
Colas máximas (aeronaves) & 18 & 13 & 5 \\
\hline Fuente: elaboración propia & &
\end{tabular}

Tabla 7. Demoras y líneas de espera en el AICM en octubre de 2015, durante el periodo de mayor actividad (entre las 6 y 24 horas) 
de estos costos para los servicios de media y alta velocidad, respectivamente.

Hay importantes beneficios ambientales con tendencia creciente de los servicios ferroviarios, en comparación con el servicio aéreo, debido a la disminución de emisiones de $\mathrm{CO}_{2}$. Por ejemplo, para 2016 con los trenes de media velocidad se estima un reducción de emisiones de $232.29 \mathrm{kt}$, y para los de alta de $274.27 \mathrm{kt}$. En particular, la ruta que más contribuye en la reducción de emisiones corresponde desde luego a la de mayor demanda (Guadalajara-México). Para esta ruta por ejemplo, en 2016, con la operación de aeronaves se generarían $161.7 \mathrm{kt}$ de $\mathrm{CO}_{2}$, en cambio con los TMV solo se generarían $31.2 \mathrm{kt}$ de $\mathrm{CO}_{2}$, lo cual representa una reducción significativa en términos absolutos (130.5 kt de $\mathrm{CO}_{2}$ ) y relativos $(80.7 \%)$.

Sin la operación de los servicios ferroviarios, el AICM alcanzará un nivel de operación crítico en octubre de 2015 (flecha A en la figura 4). Sin embargo, se estimó que en el mejor de los casos, cuando $100 \%$ de la demanda aérea cambie al modo ferroviario con los trenes de media velocidad, esta condición se presentará hasta diciembre de 2019, y con los de alta velocidad en octubre de 2019. En general, bajo esta condición el AICM alcanzará un nivel de operación crítico a finales de 2019 (flecha D en la figura 4). Por lo tanto, la implementación del servicio de trenes podría retrasar el efecto de congestión crítico en cuatro años. Aún, en el caso de otros escenarios menos favorables, en donde solo 75 o $50 \%$ de la demanda aérea se transfiere a los trenes, el retraso en la congestión total del AICM puede ser de tres o dos años, respectivamente (escenarios intermedio y pesimista, flechas C y B, respectivamente, en la figura 4).

De acuerdo con los resultados del modelo de simulación, la entrada en operación de los servicios ferroviarios con potencial generaría beneficios en la operación del AICM al sustraer parte de la demanda. Por ejemplo, en 2015 habría una reducción en las demoras y colas promedio de las aeronaves en las pistas de dos minutos y dos aeronaves, respectivamente, y en sus valores máximos de seis minutos y cinco aeronaves, respectivamente.

Cabe señalar que este estudio solo consideró el potencial de la demanda de los flujos de pasajeros aéreos, y para complementarlo se recomienda que en futuras líneas de investigación se consideren otros flujos potenciales, por ejemplo, los que se pueden atraer del autotransporte (pasajeros de autobuses y automóviles).

\section{Conclusiones}

De acuerdo con la demanda actual y los pronósticos de los servicios aéreos, en rutas menores o iguales a
500 kilómetros, se determinó que existe potencial en México para el desarrollo de diez rutas ferroviarias de media velocidad, o de ocho de alta velocidad. Ya sea en el servicio de media o de alta velocidad, se estimó una demanda potencial mayor a cuatro millones de pasajeros anuales. Las principales rutas, que comprenden alrededor de $80 \%$ de la demanda potencial son: Guadalajara-México, México-Veracruz, México-Tampico y México-Oaxaca. La primera ruta señalada concentra aproximadamente $50 \%$ de la demanda. La mayor parte de las rutas establecidas cruzan por terrenos montañosos que incrementa notablemente el costo de su infraestructura. Se debe remarcar que los costos de la infraestructura para los servicios de alta velocidad son excesivamente altos, aproximadamente 27 veces mayores que los de media velocidad, por lo que estos últimos requieren comparativamente mucho menos recursos para su instauración. Sin embargo, para determinar la viabilidad de estos proyectos se requiere la elaboración de estados proforma y el análisis de formas posibles de financiamiento, lo cual abre una línea de investigación futura. La implementación de los servicios de trenes genera beneficios ambientales, al disminuir la cantidad de emisiones contaminantes de $\mathrm{CO}_{2}$ por pasajero-kilómetro. En este caso los trenes de alta velocidad son los que más beneficios generan. Por otro lado, debido a que la mayoría de las principales rutas con potencial tienen como origen o destino al AICM, la implementación de los servicios de trenes reduciría la demanda de servicio en dicho aeropuerto, lo cual a su vez disminuiría problemas de saturación y mejoraría el servicio (reduciendo las demoras y las líneas de espera de las aeronaves en las pistas), al menos temporalmente. Se estimó que al introducir el servicio de trenes el efecto de congestión crítico del AICM se podría aplazar al menos cuatro años más, sin comprometer su crecimiento.

\section{Agradecimientos}

Los autores agradecen el apoyo brindado por el Instituto Mexicano del Transporte, el Instituto Tecnológico de Orizaba y el CONACYT.

\section{Referencias}

Banco de España. Estadísticas Complementarias del Banco de España, 2012 [en línea]. Disponible en: http://www.bde.es/webbde/es/estadis/ccff/0323.pdf

Campos-Méndez J., De Rus-Mendoza G., Barrón-De Angoiti I. El transporte ferroviario de alta velocidad. Una visión económica, España, Fundación BBVA, 2009. 
European Commission. Interactions between high-speed rail and air passenger transport, European Cooperation in the field of Scientific and Technical Research (COST 318), Belgica, 1998.

Fernandez-Díaz X. El ferrocarril en Galicia, España, editorial IR INDO, 2006.

Gobierno de la República (GR). Plan Nacional de Desarrollo 20132018, Estados Unidos Mexicanos, 2013, México, pp. 80-81, 135, 140-141.

Gutiérrez P.J. El tren de alta velocidad y sus efectos espaciales. Investigaciones Regionales-Asociación Española de Ciencia Regional, México, 2004.

Herrera-García A. Modelo de simulación de operaciones aéreas en aeropuertos saturados. El caso del Aeropuerto Internacional de la Ciudad de México. Publicación Técnica, (número 365), Instituto Mexicano del Transporte, México, 2012.

Martínez-Antonio J.J. et al. Manual estadístico del sector transporte 2012, Instituto Mexicano del Transporte, México, 2012.

Muriel R.M. La reforma ferroviaria británica de 1963, Universidad de Sevilla, España, 2011.

PPIAF G.D. La reforma de los ferrocarriles: manual para mejorar el rendimiento del sector ferroviario, Washington DC., Transport Research Support, 2011 [en línea]. Disponible en: http:// www.ppiaf.org/sites/ppiaf.org/files/documents/toolkits/railways_toolkit/PDFs/Chapter\%202/WB_Railways_Toolkit_ Chapter2_vSpanish.pdf

RENFE. Informe anual RENFE 2008, resumen ejecutivo, España, 2008.
Servicios a la Navegación en el Espacio Aéreo Mexicano (SENEAM). Publicación de Información Aeronáutica (PIA), Enmienda 06/12 (397), México, 2012.

Veldhuis J. Impact of liberalization on European airports, Transportation research board meeting, Washington, DC, USA, 1990.

Widmer J. y Axhausen K. Do rail stations at airports allow a better distribution of passenger demand among airports? 80th Annual Meeting of the Transportation Research Board. Institut für Verkehrsplanung, Transporttechnik, Strassen-und Eisenbahnbau. Washington, D.C., USA, 2001.

Widmer J. y Hidber C. Effects of rail stations at airports in Europe. Transportation Research Record. Journal of the Transportation Research Board, (número 1703), USA. 2000.

\section{Este artículo se cita: \\ Citación estilo Chicago}

Herrera-García, Alfonso, Orlando Sánchez-López. Estimación del potencial de los trenes de media y alta velocidad en México con base en la demanda de pasajeros aéreos. Ingeniería Investigación y Tecnología, XVI, 02 (2015): 265-277.

\section{Citación estilo ISO 690}

Herrera-García A., Sánchez-López O. Estimación del potencial de los trenes de media y alta velocidad en México con base en la demanda de pasajeros aéreos. Ingeniería Investigación y Tecnología, volumen XVI (número 2), abril-junio 2015: 264-277.

\section{Semblanzas de los autores}

Alfonso Herrera-García. Es ingeniero en aeronáutica por el Instituto Politécnico Nacional. Estudió la maestría en sistemas de transporte y distribución de carga en la Universidad Autónoma de Querétaro (UAQ); y el doctorado en ingeniería con mención honorífica por la UAQ. Durante trece años ha laborado en la industria de la aviación. Es profesor en la Facultad de Ingeniería de la UAQ y autor de numerosos artículos y publicaciones. En los últimos dieciséis años ha trabajado en el Instituto Mexicano del Transporte, en donde actualmente es investigador titular C"A". Su principal línea de investigación es el transporte aéreo.

Orlando Sánchez López. Es ingeniero industrial (2011) y maestro en ingeniería industrial (2014), por el Instituto Tecnológico de Orizaba (ITO). Ha participado como ponente en congresos nacionales y desarrolló la tesis de maestría en el Instituto Mexicano del Transporte. Su principal línea de trabajo se relaciona con el análisis estadístico y con las herramientas administrativas de calidad. 